\title{
On the two-stage operation mechanism of low-pressure microstrip gas chambers
}

\author{
A. Breskin, E. Shefer, R. Chechik, and A. Pansky \\ Department of Particle Physics \\ The Weizmann Institute of Science \\ 76100 Rehovot, Israel
}

\begin{abstract}
A two-stage multiplication mechanism has been observed in microstrip gas chambers (MSGC) operated in a pressure range of 10-50 Torr of isobutane. The resulting high gain $\left(>10^{4}\right)$, of single electrons photo-produced on a CsI photocathode, is attributed to a preamplification in the gas gap followed by anode strip multiplication. The large and fast rise of the induced pulse in this mode, leads to an efficient single electron detection at relatively low charge gain. The reduced positive ion feedback preserves radiation convertors coupled to such electron multipliers from sputtering damage. MSGCs operated in this mode are expected to have a subnanosecond time resolution and very high rate capability. Some potential applications are briefly discussed.
\end{abstract}

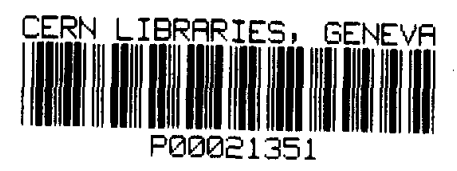

Submitted to Nuclear Instruments and Methods in Physics Research A 
We report here on some first observations of a two-stage, high gain operation of microstrip gas chambers (MSGC) at low gas pressures. Since their introduction by Oed [1] MSGCs have been subject to intense investigations [2,3]. They may provide an interesting alternative to wire chambers in many fields of application, principally due to their simplicity and high rate capability. Furthermore, they compete with silicon microstrip detectors in resolution and have a superior radiation hardness. Their ability to operate in a stable way at radiation flux superior to $1 \mathrm{MHz} / \mathrm{mm}^{2}$ [4] is derived from the fast ion removal following the charge multiplication on the anode strips, which considerably reduces space charge effects. Contrary to wire chambers, where avalanche-induced positive ions slowly drift towards the cathode planes, here, the ions are rapidly collected by the cathode strips situated a few hundred microns aside the anode strips. Due to the particular electric field distribution, only a small fraction of the avalanche-induced positive ions back-drift to the cathode plane situated above the strip electrode [5]. Therefore, this would be a major reason for combining such multiplication elements with cathode planes acting as radiation convertors, some of which could suffer from the ion sputtering [6]. Indeed, a reduction in the quantum efficiency of CSI UV-photocathodes was reported after ion bombardment in a parallel plate chamber operated at high gain [7]. In some applications, like UV, X-ray or particle imaging based on secondary electron emission from proper convertors, the gas multiplier should have high sensitivity down to single electrons, and therefore sufficiently high gain. That of MSGCs operated at atmospheric pressure is usually limited to some $10^{3}$.

We therefore proposed to operate MSGCs combining photoconvertors, at low gas pressures [8], foreseeing gain increase. This should result from a preamplification of the charges released from the convertor, under the high reduced electric field $(E / p)$ across the convertor-to-strip electrode gap. The avalanche is further multiplied at the anode strips. This fast two-stage multiplication mechanism is very similar to the one occurring in MWPCs operating at low gas pressures [9]. The main benefits, as compared to other low-pressure high gain gaseous electron multipliers $[9,10]$, being the considerably reduced positive ion feedback discussed above and the steeper current rise which is of prime im- 
portance for signal processing.

We have carried on a systematic study of the two-stage multiplication mode in lowpressure MSGCs. We employed two types of microstrip electrodes, one with $1 \mathrm{~mm}$ anode strip spacing ( $8 \mu \mathrm{m}$ wide anode, $400 \mu \mathrm{m}$ wide cathode) and a second one with $200 \mu \mathrm{m}$ spacing ( $8 \mu \mathrm{m}$ wide anode, $80 \mu \mathrm{m}$ wide cathode). Both structures were deposited on glass substrates with a metallized backing. The chamber cathode is situated at $3.2 \mathrm{~mm}$ from the strip electrode. It consists of a UV-transparent Suprasil-quartz plate, vacuum coated with a $5 \mathrm{~nm}$ of $\mathrm{Ni}-\mathrm{Cr}$ and $75 \mathrm{~nm}$ of CsI.

The detector is shown schematically in fig. 1. Single UV-induced photoelectrons are preamplified in the gas gap and further multiplied at the vicinity of the anode-strips. To understand this combined mechanism, the detector was also operated in a parallelplate mode (by connecting all anode and cathode strips to the same potential) and in a mode where multiplication occurs only at the anode strip level. The different modes were reached by setting the values of the potentials of the various electrodes (anode strips, cathode strips, cathode plane and back plane) at different levels, namely adjusting the electric field strength across the gas gap and between the strips. Fig. 2 shows charge pulses recorded on the anode strips, clearly indicating the differences between the three operation modes. They are reflected by the different pulse shapes. A slow $(2-3 \mu \mathrm{s})$ ion collection following a fast electron component is typical of the parallel-plate mode. A fast rise (of the order of $100 \mathrm{~ns}$ ) in the case of the strip multiplication is due to the rapid avalanche buildup and to fast ion collection at the cathode strips. In the two-stage multiplication mechanism we observe a large and fast rise resulting from the strip multiplication, followed by a low amplitude slow component, due to the contribution from the back-drifting ion fraction in the parallel-gap region. Fig. 3 shows the charge pulses recorded on the different electrodes in the two-stage mode. One can clearly observe the partition of the ion cloud between the two cathodes (strips and photocathode), reflected by the amplitudes of the fast and slow pulse components discussed above. The ratio of the amplitudes of the fast component to the slow component of the anode strip pulse, indicates that a large portion of the ions are collected by the cathode strips. We have confirmed that the amplitude of 
the fast component of the anode-strip pulse, is equal to the sum of amplitudes of the fast components of the two cathodes (this may not be obvious from the figure since calibrated amplifiers of different gains were connected here to the electrodes).

Amplification curves were measured in a DC mode, recording the anode-strip current resulting from a multiplication of UV-induced charges on the photocathode (see fig. 1). A $\mathrm{Hg}$ (Ar) lamp illuminated the counter through a set of foil-absorbers, used to tune the light flux. Fig. 4 shows curves resulting from separate parallel-plate or anode-strip multiplication. Only in the case of a parallel-plate the collection plateau was clearly observed. But since the same UV-flux was used also for the strip- and the combined-multiplication modes, the absolute gain could be easily derived from the measured current. One can see that in comparison with the well-expected high parallel-gap gain, the strip multiplication is very low in these low-pressure conditions. This results from the long electron mean-free-path between consecutive ionizing collisions, which sets a physical limit to the avalanche development at the vicinity of the strips. The lack of a clear plateau in the case of strip-multiplication is a result of the rapid increase with voltage of the electric field at the vicinity of the strips.

Measuring the amplification curves in the two-stage multiplication mode has been found to be very complex. Indeed, the total gain is dependent on the electric field strength at different detector locations and can therefore be set by various combinations of potentials at the electrodes. It is very difficult to maintain a constant field across the gap while varying the field between the strips. In order to achieve that, during each particular measurement several potentials have to be adjusted simultaneously. This was not the case in our measurements. Fig. 5 shows the two-stage multiplication curves at different field parameters. Note that as the photocathode voltage is increased, the slope of the gain curves is changing from a positive slope to a negative one. The positive slope is a result of a dominant increase in anode strip multiplication, while the negative slope is a result of a dominant decrease in gas-gap multiplication following an increase in microstrip voltage. At high cathode-strip voltages, the contribution of the anode-strip multiplication to the total gain is the largest, while at lower cathode-strip voltages the gain is maximal, but the 
strip contribution is smaller.

Our goal being to limit the back-drifting ion cloud, the task is therefore to find the appropriate parameters which would lead to the smallest gas-gap gain and the largest possible combined two-stage amplification factor. So far, the largest measured total gain is $4 \cdot 10^{4}$, at a pressure of 25 Torr, of which the strip multiplication contributes in a gain of the order of 20 .

We conclude that a reduction of more than an order of magnitude in ion sputtering probability, can be achieved compared to a parallel-plate detector operating under similar gain. We consider this to be a very important benefit of this operation mode.

The fast avalanche growth and ion drift at the vicinity of the strips induce large current pulses at relatively low charge gain. This could be a great advantage in single electron detection applications. Fig. 6 shows a fast current pulse (a rise-time of about 5 ns) of a single electron, detected in a two-stage mode, at a total gain of $4 \cdot 10^{4}$. All anode strips were connected to a fast ( 1 ns rise-time) current amplifier. We have compared such fast current pulses induced by multi-photon UV-light flashes, in the two-stage MSGC and in the parallel-plate operation modes. For the same charge gain an increase of 15 in the fast pulse amplitude was observed. At a gain of $4 \cdot 10^{4}$ no single electron pulses were seen under parallel-plate operation, while they are clearly above the noise level in the two-stage mode, as is apparent in Fig. 6.

Low pressure MWPCs [9] or multistep avalanche chambers [10] have been, so far, the natural choice for fast timing and parallax-free localization of charges emitted from cathode convertors. The latter could also, in principle, reduce ion-feedback, by employing transfer grids between the multiplication elements. In this case the feedback could be reduced simply due to the electrostatic opacity of mesh electrodes, or by applying electrical pulses to gate the electrodes [11]. However, for a reasonable signal-to-noise ratio in fast current pulses, these techniques usually require a gain of the order of $10^{6}$ to efficiently detect single electrons. The two-stage operation mode of the MSGC leads to equal performance at a significantly lower gain. Therefore, the reduction in ion-feedback is even more significant.

Radiation detectors that could benefit from the reduced ion-sputtering properties are 
gaseous photomultipliers [7] equipped with CsI and other photocathodes, secondary emission X-ray detectors $[12,13]$, imaging the large photon-induced secondary electron clouds emitted from the convertor surface, and particle detectors combining secondary electron emitters and gaseous electron multipliers $[9,14]$. One could also think of UV-imaging in $\mathrm{RICH}$ detectors based on MSGCs equipped with CsI photocathodes, but keeping in mind their limited size which is at present of the order of $100 \mathrm{~cm}^{2}$. An important application could be in the field of single electron counting [15] where the fast pulse development, reached in low-pressure MSGCs, could enhance the pulse counting efficiency.

Compared to MSGCs operated at normal gas pressures, the low-pressure mode is characterized by a higher gain and a faster avalanche buildup, which should result in better timing properties. A higher counting rate capability, $>1 \mathrm{MHz} / \mathrm{mm}^{2}$, is expected due to a lower charge density - resulting from a larger spread of the avalanche under the present operation conditions.

The applicability of the proposed method and its relative benefits are subject to further studies. We would like to draw the reader's attention that a development work under similar lines, namely coupling MSGC multipliers to CsI convertors, has been recently carried out in parallel at FNAL [16] and at TU-Munich [17]. The first is being directed towards fast detection of relativistic particles while the latter aims at UV-photon imaging.

This work was partially supported by the Foundation Mordoh Mijan de Salonique and and by the Basic Research Foundation of the Israel Academy of Sciences and Humanities. We would like to thank Drs. A. Oed and F. Sauli for the MSGC electrodes, Dr. L. Shekhtman for his collaboration in electric field computations, and Dr. S. Majewski for helpful remarks. 


\section{References}

1. A. Oed, Nucl. Instrum. and Meth. A263 (1988) 351.

2. See for example R. Bouclier, G. Million, L. Ropelewski, F. Sauli, Yu. N. Pestov and L.I. Shekhtman, Nucl. Instrum. and Meth. A332 (1993) 100 and references therein.

3. See for example F. Angelini, R. Bellazzini, A. Brez, G. Decarolis, C. Magazzu, M.M. Massai, G. Spandre and M.R. Torquati, Nucl. Instrum. and Meth. A315 (1992) 21 and references therein.

4. R. Bouclier, J.J. Florent, J. Gaudaen, G. Million, A. Pasta, L. Ropelewski, F. Sauli and L.I. Shekhtman, Nucl. Instrum. and Meth. A323 (1992) 236.

5. J.J. Florent, J. Gaudaen, L. Rapelewski and F. Sauli, Nucl. Instrum. and Meth. A329 (1993) 125.

6. J.S. Edmends, D.J. Miller and F. Barlow, Nucl. Instrum. and Meth. A273 (1988) 145.

7. V. Dangendorf, A. Breskin, R. Chechik and H. Shmidt-Böcking, Nucl. Instrum. and Meth. 308 (1991) 519.

8. A. Breskin, Summary talk at the First Workshop on RICH Detectors, Bari, Italy, June 2, 1993.

9. A. Breskin, Nucl. Instrum. and Meth. 196 (1982) 11.

10. A. Breskin and R. Chechik, Nucl. Instrum. and Meth. 227 (1984) 24.

11. A. Breskin, G. Charpak, S. Majewski, G. Melchart, G. Petersen, and F. Sauli, Nucl. Instrum. and Meth. 161 (1979) 19.

12. A. Breskin, R. Chechik, V. Dangendorf, S. Majewski, G. Malamud, A. Pansky, and D. Vartsky, Nucl. Instrum. and Meth. A310 (1991) 57.

13. I. Frumkin, A. Breskin, R. Chechik, V. Elkind and A. Notea, Nucl. Instrum. and Meth. A329 (1993) 337.

14. D.F. Anderson, S. Kwan and V. Peskov, High counting rate resistive plate chamber, Preprint Fermilab-Conf.-93/215.

15. A. Pansky, G. Malamud, A. Breskin, and R. Chechik, Nucl. Instrum. and Meth. 
A323 (1992) 294.

16. D.F. Anderson, S. Kwan, and M. Salomon, A low-pressure, MSGC operated with secondary electron emission. Preprint Fermilab-Pub.-94/029 (Jan. 31, 1994).

17. J. Friese, TU-München, private communication. 


\section{Figure Captions}

Fig. 1: A scheme of the low-pressure MSGC detector. Single UV-induced electrons are preamplified, starting at their production location, and further multiplied at the anode strip. Most avalanche-induced ions are collected by the cathode strips.

Fig. 2: Charge pulses recorded on the anode strips (a MSGC with $1 \mathrm{~mm}$ spaced strips) at 10 Torr of isobutane, at three different operation modes. The anode strips were grounded in all operation modes.

a) a parallel-plate multiplication: $V_{p c}=-900 \mathrm{~V}, V_{c}=0$ and $V_{b}=0$.

b) an anode strip multiplication only: $V_{p c}=-850 \mathrm{~V}, V_{c}=-450 \mathrm{~V}$, and $V_{b}=-380 \mathrm{~V}$.

c) a two-stage mode: $V_{p c}=-1045 \mathrm{~V}, V_{c}=-410 \mathrm{~V}, V_{a}=0 \mathrm{~V}$ and $V_{b}=-395 \mathrm{~V}$.

Fig. 3: The pulse-shape of charge pulses recorded on the different electrodes of the $1 \mathrm{~mm}$ spaced anode-strip MSGC in the two-stage multiplication mode, at 10 Torr of isobutane. $V_{p c}=-1020 \mathrm{~V}, V_{c}=-410 \mathrm{~V}, V_{a}=0$ and $V_{b}=-295 \mathrm{~V}$. Note that different amplifiers were connected to each electrode and therefore the pulseheights should not be compared.

Fig. 4: Single electron amplification curves, measured in a) anode-strip and b) parallelplate multiplication modes, at 10 and 25 Torr of isobutane. The MSGC has a $0.2 \mathrm{~mm}$ anode strip spacing.

Fig. 5: Single electron amplification curves in a two-stage multiplication mode at different CsI photocathode voltages. Isobutane, 25 Torr, $0.2 \mathrm{~mm}$ anode strip spacing.

Fig. 6: A single electron pulse recorded at the anode strips in a two-stage multiplication mode, using a fast ( $1 \mathrm{~ns}$ rise-time) amplifier. The charge gain is $4 \cdot 10^{4}$. Isobutane 25 Torr; $V_{p c}=-1450 \mathrm{~V}, V_{b}=V_{c}=-200 \mathrm{~V}, V_{a}=0 \mathrm{~V}$. MSGC with $0.2 \mathrm{~mm}$ anode-strip spacing. (Tektronix TDS540 digitizer, $1 \mathrm{GHz}$ sampling, $500 \mathrm{MHz}$ bandwidth). 


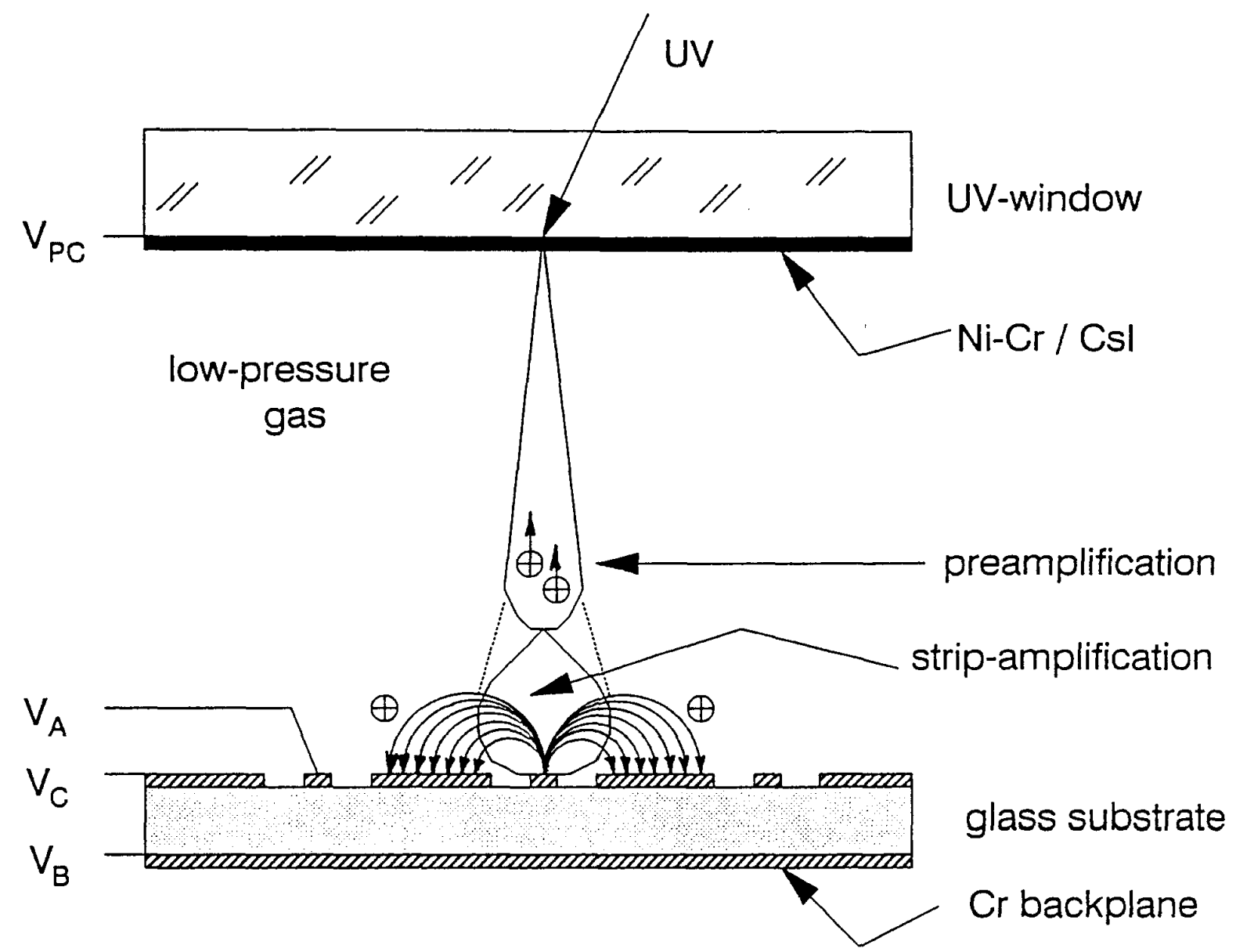

Fig. 1 

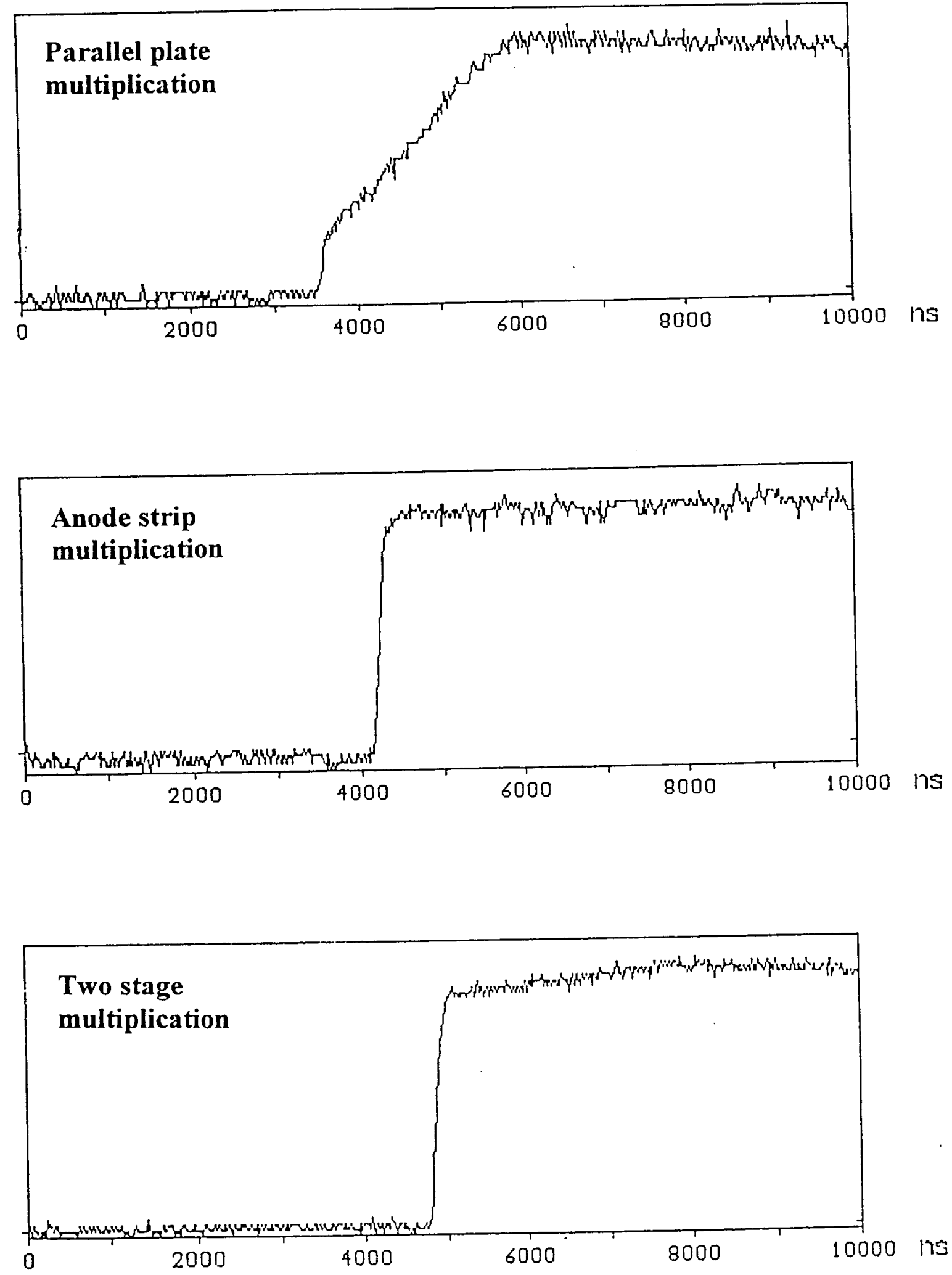

Fig. 2 

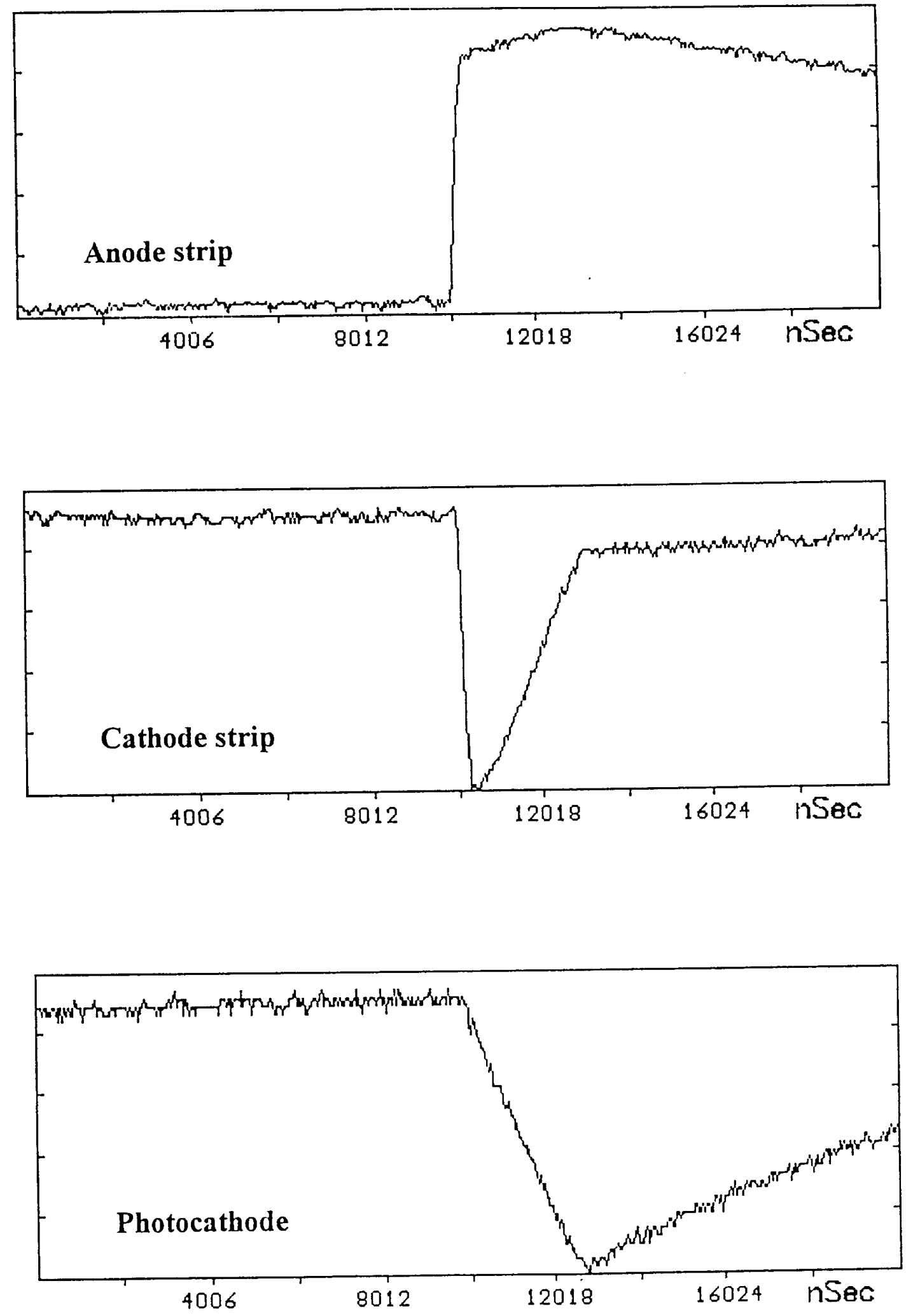

Fig. 3 

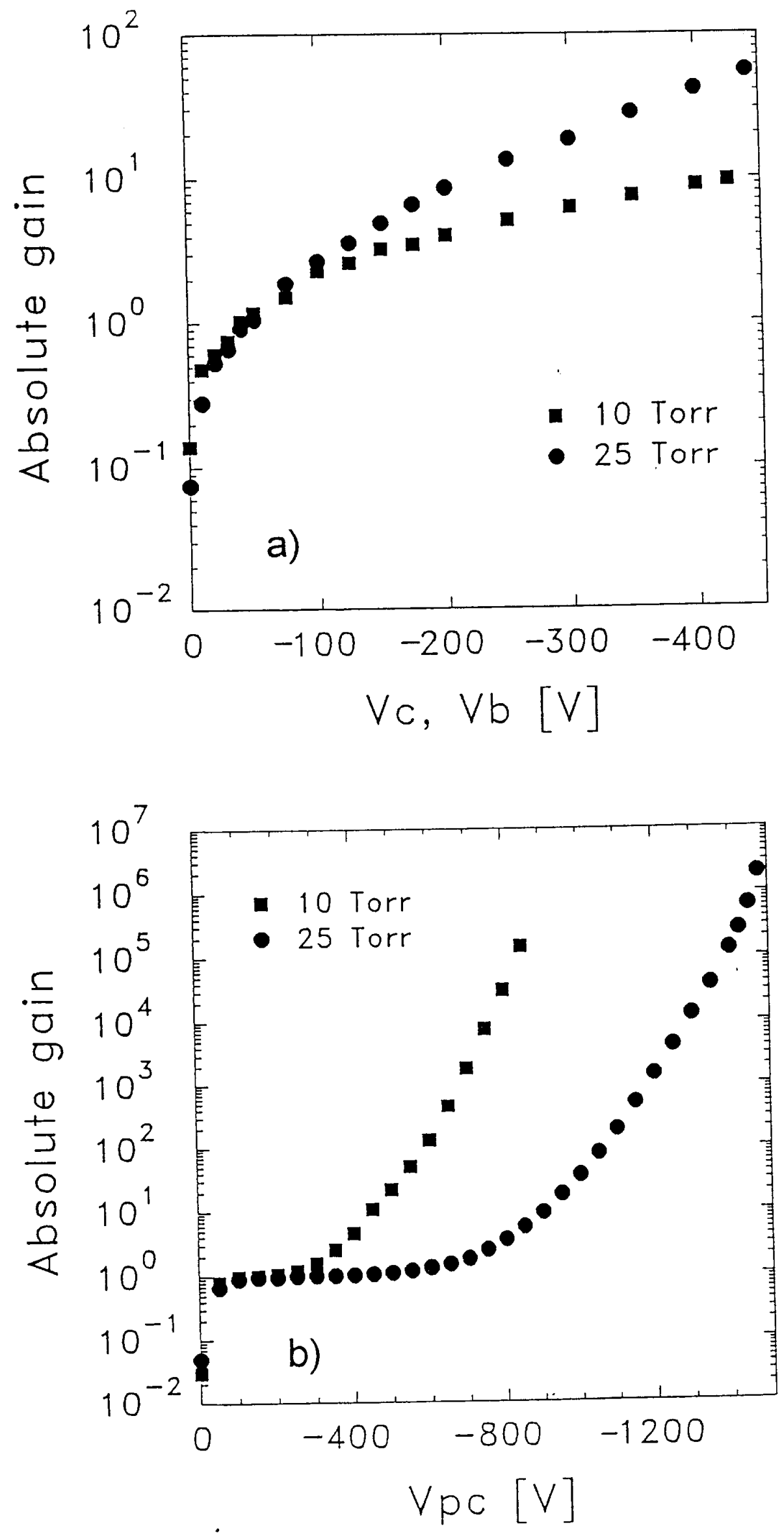

Fig. 4 


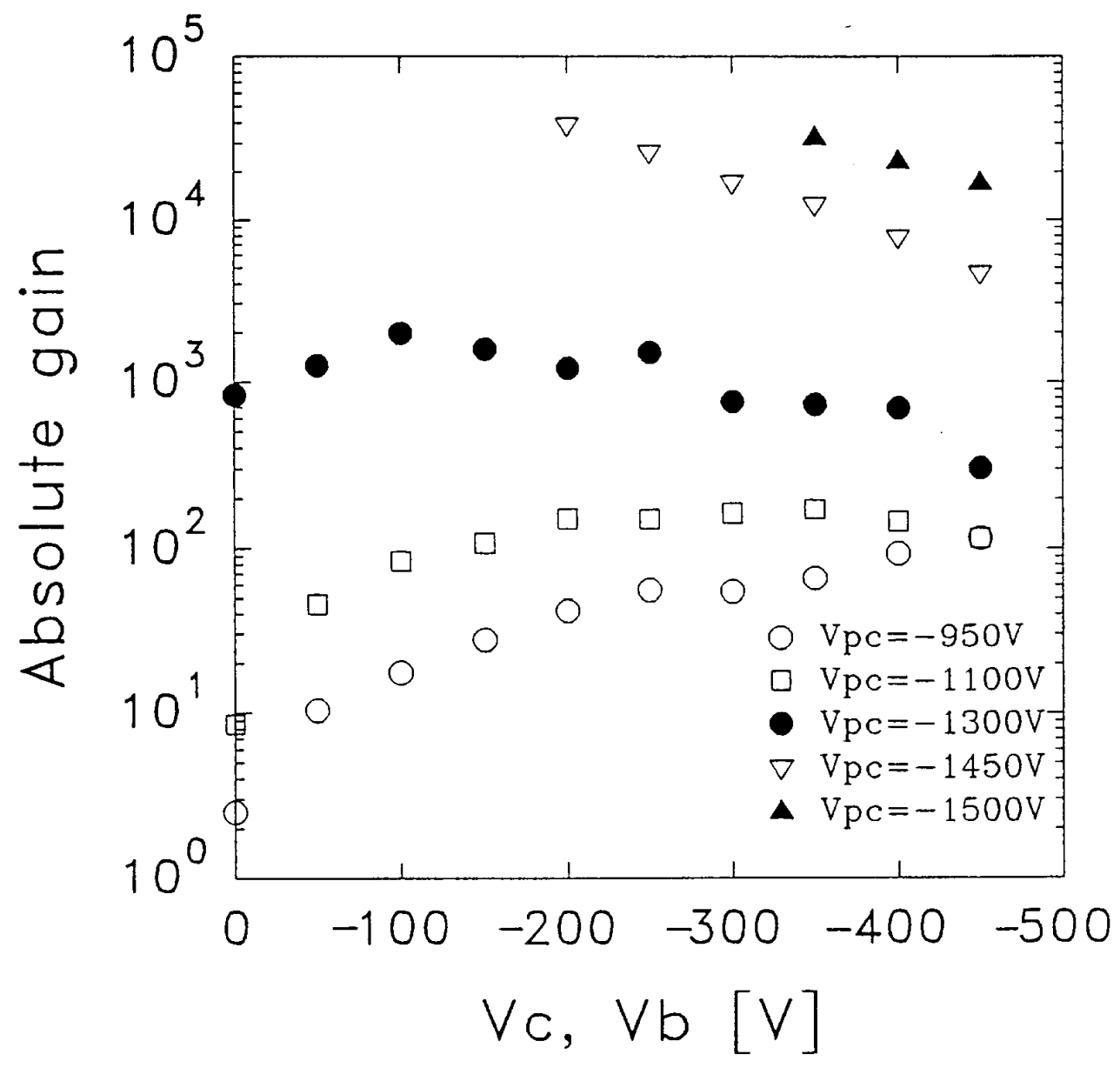

Fig. 5 


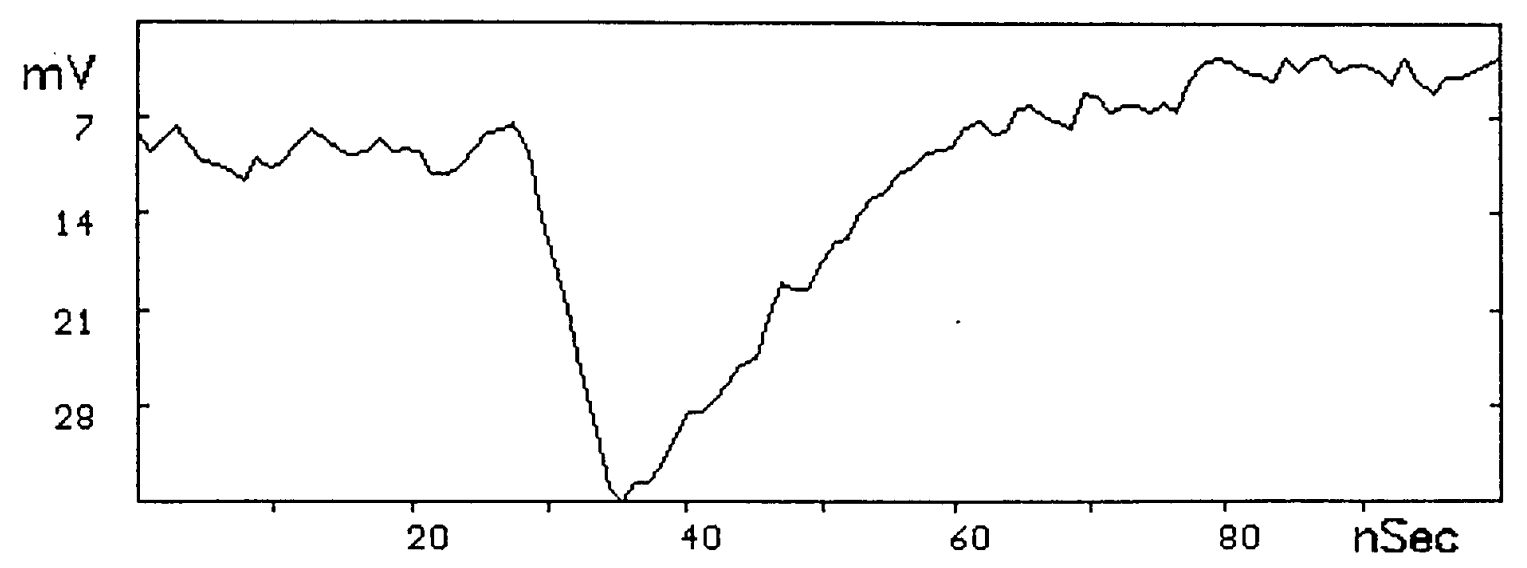

Fig. 6 